\title{
Rethinking daily aspirin for primary prevention
}

\section{An updated meta-analysis of newer RCTs seems to settle the matter as to whether to use aspirin in individuals with no known history of atherosclerotic CVD.}

\section{PRACTICE CHANGER}

Do not routinely use aspirin for primary prevention of cardiovascular disease (CVD). There is no identifiable mortality benefit for those without established CVD_regardless of risk factors. And aspirin therapy increases the risk of major bleeding.

STRENGTH OF RECOMMENDATION

A: Based on a meta-analysis of 11 randomized trials involving 157,248 patients who received aspirin for primary prevention. ${ }^{1}$

Mahmoud AN, Gad MM, Elgendy AY, et al. Efficacy and safety of aspirin for primary prevention of cardiovascular events: a meta-analysis and trial sequential analysis of randomized controlled trials. Eur Heart J. 2019;40:607-617.

\section{ILLUSTRATIVE CASE}

A 55-year-old man with well-controlled diabetes, hypertension, and sleep apnea arrives at your office for a routine annual physical. In reviewing his medications, you note that he takes a low-dose aspirin daily for "heart health." He has no known cardiovascular disease (CVD). His calculated 10 -year risk of a major cardiovascular event is $11 \%$.

Should this patient continue taking a daily aspirin for primary prevention of CVD?

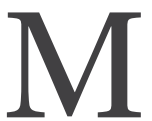
any patients in the United States take aspirin for primary prevention of CVD as recommended by the US Preventive Services Task Force (USPSTF). ${ }^{2}$ This recommendation was based on older studies of populations in which smoking rates were higher and statin use was less common, leading to an overall higher risk of CVD. ${ }^{3}$ (The USPSTF is currently in the process of updating its recommendation.) More recent RCTs have been done in patients with a lower baseline risk of CVD, and these outcomes are more generalizable to today's population. This new meta-analysis includes recent RCTs that evaluated whether there is value in using aspirin for the primary prevention of CVD.

\section{STUDY SUMMARY}

\section{No reduction in risk, increased chance of bleeding}

Mahmoud and colleagues conducted a meta-analysis of 11 randomized controlled trials that included 157,248 patients and assessed the efficacy and safety of aspirin for primary prevention of cardiovascular events. ${ }^{1}$ The mean age of the total population was 61.3 years; $52 \%$ were women and $14 \%$ were smokers. The doses of aspirin used in most of the studies were $\leq 100 \mathrm{mg} / \mathrm{d}$, although 2 of the studies examined doses that were higher. Patients were followed for a mean of 6.6 years. The primary efficacy outcome was all-cause mortality, and the primary safety outcome was major bleeding (as defined by each study). The secondary outcomes included cardiovascular mortality, fatal and nonfatal myocardial infarction (MI), and fatal and nonfatal ischemic stroke.

Aspirin did not lower all-cause mortality
Michal Poplawski, MD; Nicole K. Nelson, DO; J. Scott Earwood, MD Dwight D. Eisenhower Army Medical Center, Fort Gordon, GA

DEPUTY EDITOR Anne Mounsey, MD Department of Family Medicine, University of North Carolina, Chapel Hill

doi: $10.12788 / \mathrm{fpp} .0092$ 


Review
of current
guidelines
and studies
regarding the
use of aspirin
for primary
prevention of
CVD shows that
the tide has
been turning
against this
practice.

Review

of current

guidelines

studies

use of aspirin

for primary

prevention of

CVD shows that

the tide has

against this

practice. (risk ratio $[\mathrm{RR}]=0.98 ; 95 \%$ confidence interval [CI], 0.93-1.02) and was associated with an increased risk of major bleeding $(\mathrm{RR}=1.47$; 95\% CI, 1.31-1.65; number needed to harm = $250)$ and intracranial hemorrhage $(\mathrm{RR}=1.33$; 95\% CI, 1.13-1.58). Aspirin also had no effect on all-cause mortality in subgroup analyses of patients with diabetes mellitus or high cardiovascular risk (10-year risk $>7.5 \%$ ). There was (again) an increased risk of major bleeding.

Aspirin had no effect on the secondary outcomes-with the exception of the incidence of $\mathrm{MI}(\mathrm{RR}=0.82 ; 95 \% \mathrm{CI}, 0.71-0.94$; number needed to treat $=333$ ). However, this outcome was associated with considerable heterogeneity $\left(\mathrm{I}^{2}=67 \%\right)$, and the reduction was no longer evident after limiting the analysis to the more recent trials.

\section{WHAT'S NEW?}

\section{Study is emblematic}

\section{of a shift away from daily aspirin}

Review of current guidelines and studies regarding the use of aspirin for primary prevention of CVD shows that the tide has been turning against this practice, but the change has been gradual. Newer studieslarge RCTs such as ARRIVE (Aspirin to Reduce Risk of Initial Vascular Events) and ASCEND (A Study of Cardiovascular Events iN Diabetes)-found no mortality benefit (all-cause or cardiovascular) from using aspirin in this context.

The USPSTF guidelines in 2016 recommended prescribing daily aspirin for adults ages 50 to 59 who have a > 10\% 10-year CVD risk and discussing with adults ages 60 to 69 the risks and benefits of daily aspirin. ${ }^{2}$

The 2019 American College of Cardiology/ American Heart Association guidelines state that aspirin should no longer be used routinely for primary prevention, given the lack of net benefit. Patients ages 40 to 70 who are not at increased risk of bleeding with a higher risk of CVD may be considered for daily lowdose aspirin. The guidelines also state that adults $>70$ years or those with increased risk of bleeding should not be started on a daily aspirin. ${ }^{4}$

\section{CAVEATS}

\section{Jury is still out regarding very high-risk patients}

While the meta-analysis by Mahmoud and colleagues makes a good case for discontinuing aspirin for primary prevention in most patients, the data do not examine in detail whether there is a benefit in patients with very high risk ( $>20 \%$ ) for atherosclerotic CVD. More studies are needed before making recommendations for that specific subgroup.

\section{CHALLENGES TO IMPLEMENTATION}

\section{Patients may not be eager}

\section{to give up an accepted practice}

Physicians have been recommending aspirin for primary prevention of CVD for decades and many patients, who purchase aspirin themselves, are vested in the notion that aspirin protects them. It will take time for this change in practice to be accepted. Some patients may continue taking aspirin despite recommendation to stop. Primary care physicians will need to educate patients and clearly explain the rationale for stopping daily aspirin.

JFP

\section{ACKNOWLEDGEMENT}

The PURLs Surveillance System was supported in part by Grant Number UL1RR024999 from the National Center for Research Resources, a Clinical Translational Science Award to the University of Chicago. The content is solely the responsibility of the authors and does not necessarily represent the official views of the National Center for Research Resources or the National Institutes of Health.

Copyright $\odot$ 2020. The Family Physicians Inquiries Network. All rights reserved.

\section{REFERENCES}

1. Mahmoud AN, Gad MM, Elgendy AY, et al. Efficacy and safety of aspirin for primary prevention of cardiovascular events: a metaanalysis and trial sequential analysis of randomized controlled trials. Eur Heart J. 2019;40:607-617.

2. Bibbins-Domingo K, U.S. Preventive Services Task Force. Aspirin use for the primary prevention of cardiovascular disease and colorectal cancer: U.S. Preventive Services Task Force Recommendation Statement. Ann Intern Med. 2016;164:836-845.

3. Antithrombotic Trialists Collaboration, Baigent C, Blackwell L, Collins $\mathrm{R}$, et al. Aspirin in the primary and secondary prevention of vascular disease: collaborative meta-analysis of individual participant data from randomized controlled trials. Lancet. 2009;373:1849-1860.

4. Arnett DK, Blumenthal RS, Albert MA, et al. 2019 ACC/AHA Guideline on the Primary Prevention of Cardiovascular Disease: a report of the American College of Cardiology/American Heart Association Task Force on Clinical Practice Guidelines. J Am Coll Cardiol. 2019;74:e177-e232. 